\title{
Synthesis, Spectroscopy and X-Ray Crystal Structure of 9-Methyl-3-Thiophen-2-Yl-Thieno [3, 2-e] [1, 2, 4] Triazolo [4, 3-c] Pyrimidine-8-Carboxylic Acid Ethyl Ester
}

\author{
Nikhath Fathima ${ }^{1}$, Mohamed Ziaulla ${ }^{1}$, Afshan Banu ${ }^{1}$, Shridhar Ishwar Panchamukhi ${ }^{2}$, \\ Imtiyaz Ahmed Khazi ${ }^{2}$, Noor Shahina Begum ${ }^{{ }^{*}}$ \\ ${ }^{1}$ Department of Chemistry, Bangalore University, Bangalore, India \\ ${ }^{2}$ Department of Chemistry, Karnatak University, Dharwad, India \\ E-mail: noorsb@rediffmail.com
}

Received October 19, 2010; revised April 6, 2011; accepted May 20, 2011

\begin{abstract}
The Preparation of 9-Methyl-3-thiophen-2-yl-thieno [3, 2-e] [1, 2, 4] triazolo [4, 3-c] pyrimidine-8-carboxylic acid ethyl ester is described. Elemental analysis, IR spectrum, ${ }^{1} \mathrm{H}$ NMR, ${ }^{13} \mathrm{C}$ NMR and $\mathrm{X}$-ray crystal structure analyses were carried out to determine the composition and molecular structure of the title compound. There are two independent molecules in the asymmetric unit exhibiting intermolecular $\mathrm{C}-\mathrm{H} . . . \mathrm{N}, \mathrm{C}-\mathrm{H} . . . \mathrm{O}$ interactions with additional $\pi-\pi$ interaction that further helps in stabilizing the supramolecular structure. The results showed that the proposed method for synthesis is simple, precise and accurate which was further confirmed by crystal structure analysis.
\end{abstract}

Keywords: Thieno-Triazolo-Pyrimidine Derivative, Characterization, X-Ray Crystallographic Analysis, $\mathrm{C}-\mathrm{H} . . . \mathrm{N}, \mathrm{C}-\mathrm{H} . . . \mathrm{O}$ and $\pi-\pi$ Weak Interactions.

\section{Introduction}

Pyrimidine and thienopyrimidine derivatives are found in a variety of natural products (viz., purines, pyrrolopyrimidines, pyridopyrimidines, pteridines), agrochemicals and veterinary products $[1,2]$. Compounds containing fused pyrimidine ring make up a broad class of heterocycles that has attracted attention in the past few years owing to its wide range of biological activities such as anticancer [3], antiviral [4], antitumor [5] and anti-inflammatory activities [6]. Also, the rapid growth in the literature dealing with the synthesis and biological activity of the thienopyrimidine derivatives [7] prompted us to undertake the synthesis of novel fused thienopyridine derivative.

The synthesis of the compound was followed by measurement of the analytical data and subsequent spectroscopic analysis using IR, ${ }^{1} \mathrm{H}-\mathrm{NMR}$ and ${ }^{13} \mathrm{C}-\mathrm{NMR}$ techniques to confirm the presence of the supposed ring system. The compound was subjected to single crystal X-ray diffraction analysis so that its supramolecular structure could be investigated in terms of possible intermolecular interactions.

\section{Experimental Section}

\subsection{Materials}

All chemicals were obtained from a commercial source and used without further purification. Yellow colour single-crystals suitable for X-ray diffraction were obtained by slow evaporation of the solvents.

\subsection{Analytical Methods}

Melting points were determined in open capillaries and are uncorrected. The IR spectra were recorded on Nicolet Impact $410 \mathrm{FT}$ IR spectrophotometer using $\mathrm{KBr}$ pellets. ${ }^{1} \mathrm{H}$ and ${ }^{13} \mathrm{C}$ NMR were recorded on Bruker 300-MHz FT NMR spectrometer in $\mathrm{CDCl}_{3}$ with TMS as internal standard. Mass spectra were recorded on Finnigan MAT (Model MAT 8200) spectrometer and elemental analyses were carried out using Heraus CHN rapid analyzer.

\subsection{Preparation of 9-Methy l-3-Thiophen-2-Yl- Thieno [3, 2-e] [1, 2, 4] Triazolo [4, 3-c] Py- rimidine-8-Carboxylic Acid Ethyl Ester}

A mixture of 2 (0.2667 g, 1 mmole) and thiophene-2- 
carboxylic acid hydrazide (0.1421 g, 1 mmole) was stirred at room temperature in toluene $(5 \mathrm{ml})$ and acetic acid ( $0.060 \mathrm{~g}, 1 \mathrm{mmole}$ ) were added and the resulting reaction mixture was refluxed for $12 \mathrm{~h}$. After the completion of the reaction the reaction mixture was washed with water and dried over sodium sulphate. Toluene was removed under reduced pressure to obtain analytically pure compound.

\subsection{Physical Measuments: 9-Methyl-3-Thiophen -2-Yl-Thieno[3, 2-e][1, 2, 4]Triazolo[4, 3-c] Pyrimidine-8-Carboxylic Acid Ethyl Ester}

Yield $75 \%$, mp: $174^{\circ} \mathrm{C}-176^{\circ} \mathrm{C}$. IR $(\mathrm{KBr}) v \cdot \mathrm{cm}^{-1}$ : 3068, 2983, 2924, 1728, 1617, 1531. ${ }^{1} \mathrm{H}$ NMR (300 MHz, $\left.\mathrm{CDCl}_{3}\right): \delta 1.42\left(t, 3 \mathrm{H}, \mathrm{CH}_{3}\right), 3.2\left(s, 3 \mathrm{H}, \mathrm{CH}_{3}\right), 4.47$ $\left(q, 2 \mathrm{H}, \mathrm{CH}_{2}\right), 7.29-7.94(m, 3 \mathrm{H}, \mathrm{ArH}), 9.78(s, 1 \mathrm{H}$, $\left.\mathrm{C}_{5}-\mathrm{H}\right) .{ }^{13} \mathrm{C}$ NMR (75 MHz, $\mathrm{CDCl}_{3}$ ): $\delta$ 14.7, 15.6, 62.0, $121.8,128.2$, 128.4, 129.3, 129.6, 132.7, 138.5, 140.6, 150.5, 156.3, 161.6, 162.6. Anal. calcd. for $\mathrm{C}_{15} \mathrm{H}_{12} \mathrm{~N}_{4} \mathrm{O}_{2}$ S: C, 52.31; H, 3.51; N, 16.27; S, 18.62 Found: C, 52.88; H, 3.80; N, 16.32; S,18.42. MS m/z: $346.07(m+2)$.

\section{X-Ray Diffraction Analysis}

The X-ray diffraction data for the compound 3 was collected on a Bruker Smart CCD Area Detector System, using MoK $\alpha$ (0.71073 $\AA)$ radiation for the crystal. Intensity data were collected up to a maximum of $29.41^{\circ}$ in the $\omega-\phi$ scan mode. The data were reduced using SAINTPLUS [8]. The structure was solved by direct methods using SHELXS97 [9] and difference Fourier synthesis using SHELXL97 [9]. The positions and anisotropic displacement parameters of all non-hydrogen atoms were included in the full-matrix least-square refinement using SHELXL97 [9] and the procedure were carried out for a few cycles until convergence was reached. A total of 34420 reflections were collected, re-

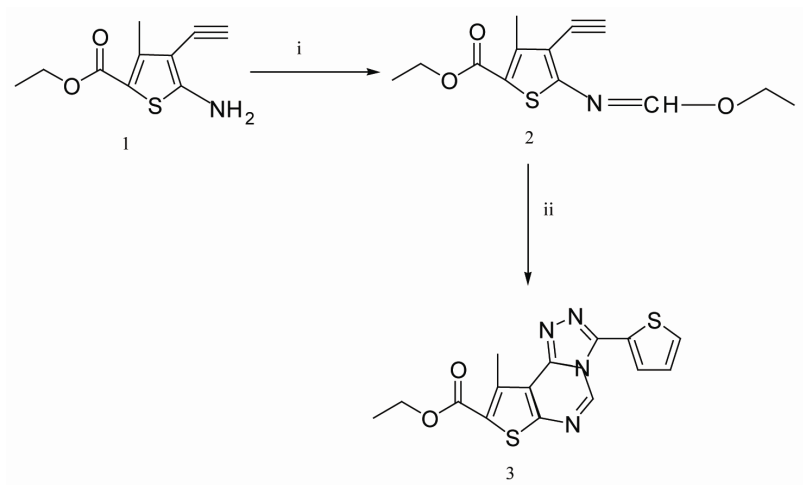

Reagent and Conditions: i) Triethylorthoformate, reflux ii) $\mathrm{RCONHNH}_{2}$, AcOH, reflux $12 \mathrm{~h}$.
Table 1. Crystal data and structure refinement

\begin{tabular}{|c|c|}
\hline Empirical formula & C15 H12 N4 O2 S2 \\
\hline Formula weight & 344.41 \\
\hline Temperature & 293(2) K \\
\hline Wavelength & $0.71073 \AA$ \\
\hline Crystal system, space group & Triclinic, P-1 \\
\hline \multicolumn{2}{|l|}{ Unit cell dimensions } \\
\hline a $(\AA)$ & $8.3986(5)$ \\
\hline b $(\AA)$ & $8.9813(6)$ \\
\hline с $(\AA)$ & 23.3149(12) \\
\hline$\alpha(\AA)$ & $83.888(5)$ \\
\hline$\beta \quad\left(^{\circ}\right)$ & 79.673(5) \\
\hline$\gamma\left({ }^{\circ}\right)$ & $62.904(7)$ \\
\hline Volume $\AA^{3}$ & $1539.65(2)$ \\
\hline $\mathrm{Z}$ & 4 \\
\hline Calculated density $\left(\mathrm{mg} / \mathrm{m}^{3}\right)$ & 1.486 \\
\hline Absorption coefficient $\left(\mathrm{mm}^{-1}\right)$ & 0.360 \\
\hline $\mathrm{F}(000)$ & 712 \\
\hline Crystal size & $0.4 \mathrm{~mm} \times 0.35 \mathrm{~mm} \times 0.3 \mathrm{~mm}$ \\
\hline Theta range for data collection & $2.55-29.41$ \\
\hline Limiting indices & $-11<=\mathrm{h}<=11$ \\
\hline & $-12<=\mathrm{k}<=12$ \\
\hline & $-30<=1<=31$ \\
\hline Reflections collected / unique & $34420 / 7623[\mathrm{R}(\mathrm{int})=0.0726]$ \\
\hline Completeness to theta & $29.4189 .3 \%$ \\
\hline Refinement method & Full-matrix least-squares on $\mathrm{F}^{2}$ \\
\hline Data/restraints/parameters & $7623 / 0 / 425$ \\
\hline Goodness-of-fit on $\mathrm{F}^{2}$ & 0.934 \\
\hline Final $\mathrm{R}$ indices [I > 2sigma(I)] & $\mathrm{R} 1=0.0821, \mathrm{wR} 2=0.2136$ \\
\hline $\mathrm{R}$ indices (all data) & $\mathrm{R} 1=0.1341, \mathrm{wR} 2=0.2383$ \\
\hline Largest diff. peak and hole(e Å-3) & 0.851 and -0.196 \\
\hline
\end{tabular}

sulting in $7623[\mathrm{R}(\mathrm{int})=0.0726]$ independent reflections of which the number of reflections satisfying $\mathrm{I}>2 \sigma(\mathrm{I})$ criteria were 4579. These were treated as observed. The $\mathrm{H}$ atoms were placed at calculated positions in the riding model approximation (C-H $0.93 \AA$ ), with their tem- perature factors were set to 1.2 times those of the equivalent isotropic temperature factors of the parent atoms. All other non- $\mathrm{H}$ atoms were refined anisotropi- cally. The $\mathrm{R}$ factor for observed data finally converged to $\mathrm{R}=0.0821$ with $\mathrm{wR}_{2}=0.2136$ in the compound. The maximum and minimum values of residual electron den- sity were 0.851 and $-0.196 \mathrm{e}^{-3}$. Molecular diagrams were generated using ORTEP [10]. The mean plane cal- culation was done using the program PARST [11].

\section{Results and Discussion}

\subsection{Chemistry}

The required 5-amino-4-cyano-3-methylthiophene-2-carboxylic acid ethyl ester 1 was prepared by Gewald's reaction as reported in the literature [12] Formation of 5amino-4-cyano-3-methylthiophene-2-carboxylic acid ethyl ester 1 was characterized by the presence of band at $2210 \mathrm{~cm}^{-1}$ due to cyano group and N-H stretching bands at 3339 and $3190 \mathrm{~cm}^{-1}$ in their IR spectrum. Further it was also supported by the presence of $\mathrm{D}_{2} \mathrm{O}$ exchangeable 
Table 2. Bond lengths [Å].

\begin{tabular}{cc}
\hline Atoms & Length \\
\hline C1-C2 & $1.494(7) / 1.480(9)$ \\
C2-O1 & $1.461(6) / 1.457(6)$ \\
C3-O2 & $1.202(6) / 1.196(6)$ \\
C3-O1 & $1.330(6) / 1.325(6)$ \\
C3-C4 & $1.475(7) / 1.479(6)$ \\
C4-C5 & $1.378(6) / 1.370(7)$ \\
C4-S1 & $1.737(4) / 1.736(5)$ \\
C5-C7 & $1.417(6) / 1.437(6)$ \\
C5-C6 & $1.502(6) / 1.494(7)$ \\
C7-C8 & $1.395(6) / 1.389(6)$ \\
C7-C9 & $1.427(6) / 1.422(6)$ \\
C8-N1 & $1.387(6) / 1.384(6)$ \\
C8-S1 & $1.704(5) / 1.722(5)$ \\
C9-N4 & $1.328(6) / 1.325(6)$ \\
C9-N2 & $1.381(6) / 1.383(5)$ \\
C10-N1 & $1.280(6) / 1.283(7)$ \\
C10-N2 & $1.374(6) / 1.361(7)$ \\
C11-N3 & $1.345(6) / 1.328(6)$ \\
C11-N4 & $1.364(6) / 1.368(6)$ \\
C11-C12 & $1.452(6) / 1.447(6)$ \\
C12-C15 & $1.443(8) / 1.588(8)$ \\
C12-S2 & $1.713(5) / 1.706(5)$ \\
C13-C14 & $1.454(9) / 1.343(7)$ \\
C14-C15 & $1.338(8) / 1.43(9)$ \\
C15-S2 & $1.679(6) / 1.663(6)$ \\
N2-N3 & $1.374(5) / 1.375(5)$ \\
\hline
\end{tabular}

*The values given after/correspond to molecule B

broad singlet at $\delta 7.97$ in ${ }^{1} \mathrm{H}$ NMR spectrum due to $\mathrm{NH}_{2}$ group.

Imidoformate 2 was prepared in excellent yield by treating 1 with triethylorthoformate in refluxing temperature (scheme 1). The structure of imidoformate 2 was established by the absence of $v_{\mathrm{N}-\mathrm{H}}$ in IR and the presence of a triplet at $\delta 1.42$ and a quartet at $\delta 4.47$ corresponding to protons of the ethoxy group and peak around $\delta 8.47$ due to $\mathrm{N}=\mathrm{CH}$ in the ${ }^{1} \mathrm{H}$ NMR spectrum, along with the expected signals. The reaction of imido- formate ester 2 with thiophene-2-carboxylic acid hy- drazide in refluxing toluene afforded the desired 9Methyl-3-thiophen-2-yl-thieno [3, 2-e] [1, 2, 4] triazolo [4, 3-c] pyrimidine-8-carboxylicacid ethyl ester (3) in moderate yield.

The structure of the target compound was ascertained by the analytical and spectral data. IR spectra of this compound exhibited bands at 1617 and 1531 due to

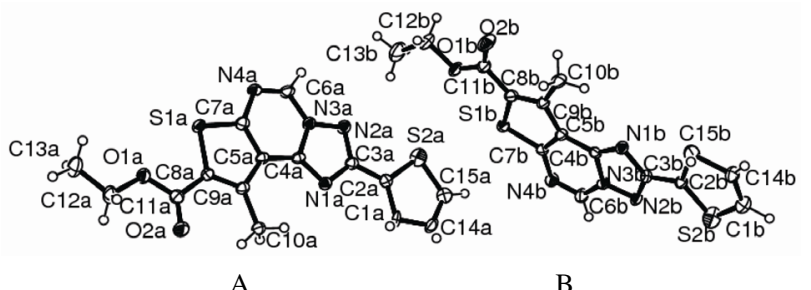

Figure 1. ORTEP diagram of compound (2), showing $50 \%$ probability displacement ellipsoids and the atom-numbering scheme.
Table 3. Selected bond angles $\left[{ }^{\circ}\right]$.

\begin{tabular}{|c|c|}
\hline Atoms & Angle \\
\hline O1-C2-C1 & $107.3(4) / 108.2(5)$ \\
\hline O2-C3-O1 & $124.1(5) / 124.0(4)$ \\
\hline $\mathrm{O} 2-\mathrm{C} 3-\mathrm{C} 4$ & $124.9(4) / 124.2(5)$ \\
\hline O1-C3-C4 & $111.0(4) / 111.7(4)$ \\
\hline C5-C4-C3 & $127.8(4) / 127.3(4)$ \\
\hline C5-C4-S1 & 113.8(3)/114.3(3) \\
\hline C3-C4-S1 & $118.3(3) / 118.4(4)$ \\
\hline C4-C5-C7 & $110.1(4) / 110.0(4)$ \\
\hline C4-C5-C6 & $126.9(4) / 126.8(4)$ \\
\hline C7-C5-C6 & $123.1(4) / 123.1(4)$ \\
\hline C8-C7-C5 & $113.4(4) / 113.2(4)$ \\
\hline C8-C7-C9 & $115.4(4) / 115.6(4)$ \\
\hline N1-C8-C7 & $126.0(4) / 127.0(4)$ \\
\hline N1-C8a-S1 & $121.7(3) / 120.8(3)$ \\
\hline C7-C8-S1 & $112.3(3) / 112.2(3)$ \\
\hline N4-C9-N2 & $109.6(4) / 109.9(4)$ \\
\hline N4-C9-C7 & $134.3(4) / 135.0(4)$ \\
\hline N2-C9-C7 & $116.0(4) / 115.1(4)$ \\
\hline N1-C10-N2 & $122.2(4) / 123.1(5)$ \\
\hline N3-C11-N4 & $115.9(4) / 116.2(4)$ \\
\hline N3-C11-C12 & $122.8(4) / 122.0(4)$ \\
\hline N4-C11-C12 & $121.3(4) / 121.8(4)$ \\
\hline C11-C12-S2 & $122.8(3) / 122.0(4)$ \\
\hline C13-C12-S2 & $113.1(4) / 118.8(4)$ \\
\hline C12-C13-C14 & $106.9(6) / 107.9(6)$ \\
\hline C15-C14-C13 & $114.2(6) / 116.3(6)$ \\
\hline C14-C15-S2 & $114.4(5) / 114.7(5)$ \\
\hline C3-O1-C2 & $116.0(4) / 116.7(4)$ \\
\hline C10-N1-C8 & $116.5(4) / 114.8(4)$ \\
\hline C10-N2-N3 & $126.1(4) / 126.4(4)$ \\
\hline C10-N2-C9 & $123.9(4) / 124.4(4)$ \\
\hline N3-N2-C9A & $110.0(3) / 109.2(4)$ \\
\hline C11-N3-N2 & $101.3(4) / 101.9(3)$ \\
\hline C9-N4-C11 & $103.1(4) / 102.8(4)$ \\
\hline C8-S1-C4 & $90.5(2) / 90.3(2)$ \\
\hline C15-S2-C12 & $91.4(3) / 91.9(3)$ \\
\hline
\end{tabular}

*The values given after/correspond to molecule B

$\mathrm{C}=\mathrm{N}$ and $\mathrm{C}=\mathrm{C} .{ }^{1} \mathrm{H}$ NMR spectra displayed the absence of peaks due to ethoxy protons present in compounds 2 and presence of thiophenic protons substituent in 3. Finally the structures were confirmed by their mass spectral data and single crystal $\mathrm{x}$-ray diffraction analysis.

\subsection{Crystallography}

Summary of crystallographic data and other structure refinement parameters of the title compound are shown in Table 1. The selected bond lengths and bond angles of all the non-hydrogen atoms are given in Tables 2 and Table 3. Table 4 shows the respective hydrogen bond interactions of the compound. The ORTEP view of the molecule with atomic labeling (thermal ellipsoids drawn at 50\% probability) is shown in Figure 1. Figures 2 and $\mathbf{3}$ show the packing of molecules in the crystal structure. Figure 4 shows $\pi$ - $\pi$ interaction.

The compound 3 consists of two independent molecules $\mathbf{A}$ and $\mathbf{B}$ respectively in the asymmetric unit. The tricyclic system including the condensed thiophene, pyri- 
Table 4. Non-bonded interactions and possible hydrogen bonds $\left(\AA,{ }^{\circ}\right)$ for compound 3 . (D-donor; A-acceptor; Hhydrogen).

\begin{tabular}{ccccc}
\hline D-H $\cdots A$ & D-H & H $\cdots A$ & D $\cdots A$ & D-H $\cdots A$ \\
\hline C10a-H10a...O2a & $0.960(5)$ & $2.335(5)$ & $3.052(7)$ & 131 \\
C10b-H10b2...N1b & $0.960(5)$ & $2.572(5)$ & $3.302(8)$ & 133 \\
C6b-H6b...N2b ${ }^{\mathrm{i}}$ & $0.885(5)$ & $2.584(9)$ & $3.374(9)$ & 149 \\
C14a-H14a...O2a ${ }^{\text {ii }}$ & $0.890(9)$ & $2.728(1)$ & $3.122(7)$ & 138 \\
C6a-H6a...O2a ${ }^{\text {ii }}$ & $0.885(5)$ & $2.752(2)$ & $3.248(8)$ & 116 \\
\hline
\end{tabular}

Symmetry code: 0 ) $x, y, z$; i) $-x+1,-y,-z-1$; ii) $-x+2,-y,-z$.

midine, and triazole rings is virtually planar as reported earlier [13]. The dihedral angle between the condensed thiophene-triazolo-pyrimidine rings and thieno ring is coplanar inclined at an angle $13.77^{\circ}$ and $12.76^{\circ}$ in the molecules $\mathbf{A}$ and $\mathbf{B}$ respectively. The methyl group is cis to the carboxylic acid ethyl ester group and almost orthogonal to thiophene-triazolo-pyrimidine rings at an angle $85.89^{\circ}$ and $85.21^{\circ}$ respectively.

The intramolecular interaction is different in molecules A and B. Molecule A shows C-H...O interaction whereas it is C-H...N in molecule $\mathbf{B}$. In the molecule $\mathbf{A}$, the carbonyl group has cis orientation with respect to the C8a = C9a double bond leading to a strong intramolecular C10a-H10a...O2a hydrogen bond [C10a-H10a = 0.960(5) $\AA$, H10a...O2a = 2.338(5) $\AA$, C10a...O2a = 3.056(7) $\AA$ and the angle C10a-H10a...O2a = $131^{\circ}$ ]. In the molecule $\mathbf{B}$, the strong intramolecular hydrogen bond is $\mathrm{C} 10 \mathrm{~b}-\mathrm{H} 10 \mathrm{~b} 2 \ldots \mathrm{N} 1 \mathrm{~b}$ [C10b-H10b2 = 0.960(5) $\AA$, $\mathrm{H} 10 \mathrm{~b} 2 \ldots \mathrm{N} 1 \mathrm{~b}=2.567(5) \AA, \mathrm{C} 10 \mathrm{~b} . . \mathrm{N} 1 \mathrm{~b}=3.303(8) \AA$ and the angle C10a-H10b2...N1b = 133]. These two

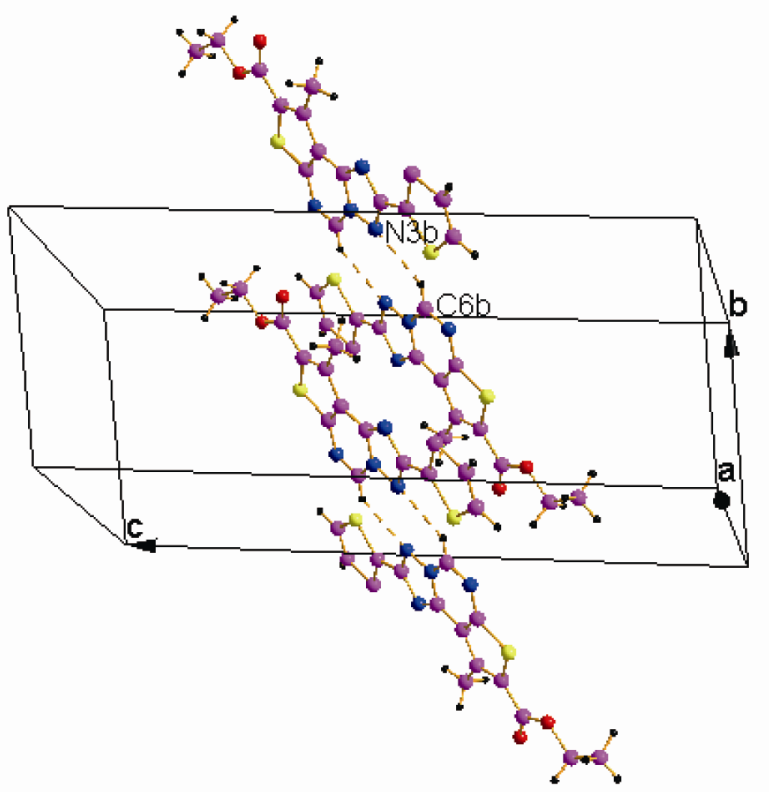

Figure 2. C-H....N intermolecular interactions viewed roughly along ' $a$ ' axis. interactions in molecules $\mathbf{A}$ and $\mathbf{B}$ leads to the formation of a pseudo-seven-membered ring pattern with graph set motif S(7) [14], thus locking the molecular conformation and eliminating conformational flexibility. The orientation of the carboxylic acid ethyl ester group is characterized by the torsion angles $\mathrm{S}(1 \mathrm{a})-\mathrm{C}(8 \mathrm{a})-\mathrm{C}(11 \mathrm{a})-\mathrm{O}(1 \mathrm{a})$ $\left[9.7(6)^{\circ}\right]$ in molecule $\mathbf{A}$ and $\mathrm{S}(1 \mathrm{~b})-\mathrm{C}(8 \mathrm{~b})-\mathrm{C}(11 \mathrm{~b})-\mathrm{O}(1 \mathrm{~b})$ $\left[3.5(1)^{\circ}\right]$ in the molecule $\mathbf{B}$ respectively.

The crystal structure of molecules $\mathbf{A}$ and $\mathbf{B}$ are stabilized by some interesting features that comprises of intermolecular interactions $\mathrm{C}-\mathrm{H} . . . \mathrm{N}$ and $\mathrm{C}-\mathrm{H} . . . \mathrm{O}$. The $\mathrm{C}-\mathrm{H}$... $\mathrm{N}$ interaction results in centrosymmetric head to head dimers corresponding to graph set motif $\mathrm{R}_{2}^{2}(8)$ (Bernstein, et al., 1995) as depicted in [Figure 2]. There are two C-H...O hydrogen bonds [Figure 3], linking the molecules in a cohesive manner. One of the C-H...O interaction forms bifurcated bond from two donors $\mathrm{C} 6 \mathrm{~b}$ and $\mathrm{C} 14 \mathrm{~b}$ to the same acceptor O2b resulting in a zig-zag pattern. The other C-H...O interaction forms centrosymmetric head to head dimers corresponding to graph

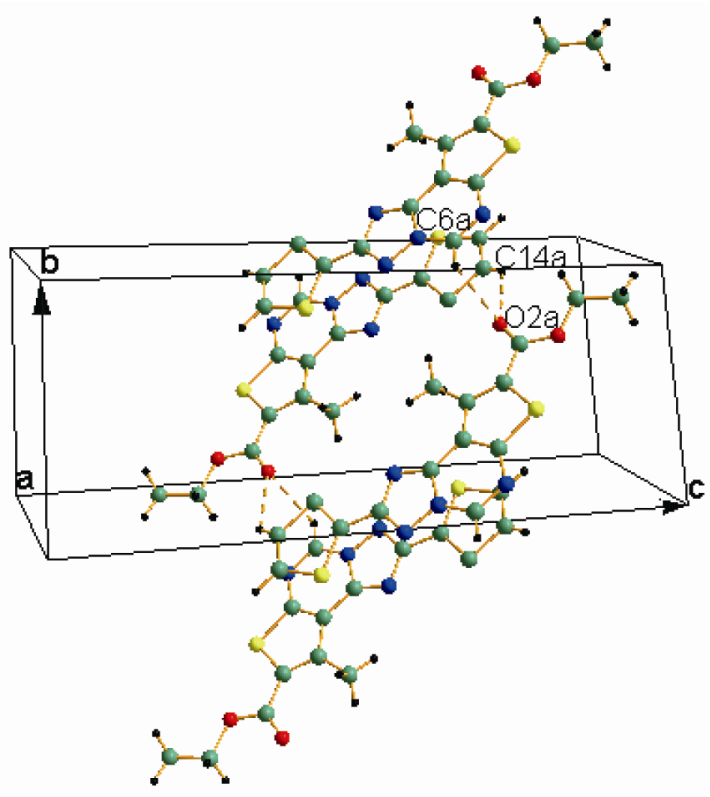

Figure 3. Packing of the molecules in crystal of (3) viewed along ' $a$ ' axis. Dotted lines indicate $\mathrm{C}-\mathrm{H}$...O intermolecular interactions generating bifurcated bond resulting in dimers.

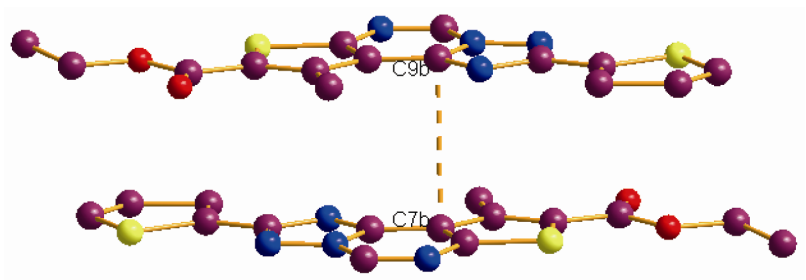

Figure 4. $\pi-\pi$ stacking interactions between the two pyrimidine rings. 
set notation $\mathrm{R}_{2}^{2}$ (24) (Bernstein et al., 1995). The $\pi-\pi$ stacking interaction [Figure 4] between the two pyrimidine rings being separated by a distance of 3.424(1) $\AA$ (symmetry code: $1-\mathrm{x}, 1-\mathrm{y},-\mathrm{z}$ ) further strengthens the supramolecular structure.

\section{Conclusions}

In this paper we report the synthesis of 9-Methyl-3- thiophen-2-yl-thieno [3, 2-e] [1, 2, 4] triazolo [4, 3-c] pyrimidine-8-carboxylic acid ethyl ester. The X-ray analysis was carried out in order to establish supramolecular assembly in terms of possible intermolecular interactions. In the present work the crystallographic studies aims at highlighting the importance of hydrogen bond interaction which is one of the most important of all directional in- termolecular interactions. Here several hydrogen bond- ing interactions of the triazolo-pyrimidine groups and the interaction between carboxylic acid ester and the pyrimi- dine group between the two different molecules have been demonstrated.

\section{Supplementary Material}

Crystallographic data for the structure (3) reported in this paper have been deposited with the Cambridge data centre. The deposition number is CCDC $\mathbf{7 8 7 3 3 5 .}$

\section{Acknowledgements}

N. S. B is thankful to the University Grants Commission (UGC), India for the financial assistance.

\section{Reference}

[1] B. Roth, C. Cheng, "Progress in Medicinal Chemistry", (Eds. G. P. Ellis and G. B. West), Elsevier Biomedical Press, New York, 1982.

[2] D. J. Brown, "Pyrimidines and their Benzo Derivatives, in Comprehensive Heterocyclic Chemistry,” (Eds. A. R. Katritzky and C. W. Rees), Pergamon Press, Oxford, 1984.

[3] C. R. Petrie, H. B. Cottam, P. A. Mckernan, R. K. Robins and G. R. Revankar, "Synthesis and Biological Activity of 6-Azacadeguomycin and Certain 3,4,6-Trisubstituted Ppyrazolo[3, 4-d]Pyrimidine Ribonucleosides,” Journal of Medicinal Chemistry, Vol. 28, No. 8, 1985, pp. 10101016. doi:10.1021/jm00146a007

[4] M. N. Nasr, M. M. Gineinah, "Pyrido[2, 3-d]Pyrimidines and Pyrimido[5',4':5,6]Pyrido[2, 3-d]Pyrimidines as New Antiviral Agents: Synthesis and Biological Activity,”
Archiv der Pharmazie Chemistry in Life Science, Vol. 335, No. 6, 2002, pp. 289-295. doi:10.1002/1521-4184(200208)335:6<289::AID-ARDP2 89>3.0.CO;2-Z

[5] P. G Baraldi, M. G Pavani, M. D. Nunez, P. Brigidi, B. Vitali, R. Gambari and R. Romanoli, "Antimicrobial and Antitumor Activity of N-Heteroimmine-1,2,3-Dithiazoles and their Transformation in Triazolo-, Imidazo-, and Pyrazolopirimidines,” Bioorganic \& Medicinal Chemistry, Vol. 10, No. 2, 2002, pp. 449-456. doi:10.1016/S0968-0896(01)00294-2

[6] S. M. Sondhi, M. Johar, S. Rajvanshi, S. G. Dastidar, R. Shukla, R. Raghubir and J. W. Lown, "Anticancer, Anti-Inflammatory and Analgesic Activity Evaluation of Heterocyclic Compounds Synthesized by the Reaction of 4-Isothiocyanato-4-Methylpentan-2-One with Substituted $o$-Phenylenediamines, $o$-Diaminopyridine and (Un) Substituted o," Australian Journal of Chemistry, Vol. 54, 2001, pp. 69-74. doi:10.1071/CH00141

[7] N. S. Shetty, R. S. Lamani and I. M. Khazi, "Synthesis and Antimicrobial Activity of Some Novel Thienopyrimidines and Triazolothienopyrimidines," Journal of Chemical Sciences, Vol. 121, No. 3, 2009, pp. 301-307. doi:10.1007/s12039-009-0034-7

[8] Bruker and SAINTPLUS, "Program for Data Reduction," Bruker Axs Inc., Madison, USA, 1998.

[9] G. M. Sheldrick, "Program for Crystal Structure Refinement," SHELXL97, University of Göttingen, Germany, 1997.

[10] L. J. Farrugia, "ORTEP-3 for WINDOWS-A Version of ORTEP-III with a Graphical User Interface (GUI)," Journal of Applied Crystallography, 1997, Vol. 30, No. 1, pp. 565-566. doi:10.1107/S0021889897003117

[11] M. Nardelli, "Ring Asymmetry Parameters from Out-ofPlane Atomic Displacements," Acta Crystallgraphcia Section C, Vol. 39, No. 8, 1983, pp. 1141-1142. doi:10.1107/S0108270183007696

[12] Z. Puterova and A. Andicsova, "A Facile Synthesis of $\alpha$-Substituted Thiophenes from a Functionalized 2-Aminothiophene by Homo- and Cross-Coupling Reactions," Tetrahedron Letters, Vol. 50, No. 32, 2009, pp. 46704673. doi:10.1016/j.tetlet.2009.06.006

[13] A. Sh. Oganisyan1, A. S. Noravyan1, A. A. Karapetyan, M. S. Aleksanyan1 and Yu. T. Struchkov, "Derivatives of Condensed Thienopyrimidimines. 13* Synthesis and Structure of Pyrano[4',3':4,5]Thieno-[3, 2-e]-1,2,4-Tria-zolo [2, 3-c]Pyrimidines," Chemistry of Heterocyclic Compounds, Vol. 37, No. 52001, pp. 628-632.

[14] J. Bernstein, R. E. Davis, L. Shimoni and N. L. Chang, "Patterns in Hydrogen Bonding: Functionality and Graph Set Analysis in Crystals," Angewandte Chemie International Edition, Vol. 34, No. 15, 1995, pp. 1555-1573. doi:10.1002/anie.199515551 